\title{
Hypernuclear Spectroscopy with Electron Beams
}

\section{Patrick ACHENBACH*}

Institut für Kernphysik, J. J. Becherweg 45,

Johannes Gutenberg-Universität, D-55099 Mainz, Germany

E-mail: patrick@kph.uni-mainz.de

\begin{abstract}
A $\Lambda$-hyperon bound to a nuclear core, forming a hypernuclei, is a unique probe of the interior of hadronic many-body systems. Only recently, the spectroscopy of $\Lambda$-hypernuclei with electron beams was realized. Today, two places worldwide offer the possibility to study hypernuclei with high intensity continuous electron beams: MAMI at Mainz, Germany, and Jefferson Lab (JLab), Virginia. At least two different techniques exist for such studies: reaction spectroscopy, which requires two high-resolution spectrometers, one for kaons and one for the scattered electrons, and decay particle spectroscopy of hyperfragments. In the latter case a strangeness tagger in forward direction is of great advantage to reduce the background in the pion spectrum. In Mainz, the first hypernuclei experiments on pion decay spectroscopy to be performed with the dedicated kaon spectrometer KAOS as strangeness tagger are scheduled for Summer 2011. At JLab, hypernuclear reaction spectroscopy with electron beams was pioneered in the the year 2000 , followed by a decade of activities that peaked with the third generation experiment E05-115 in Hall C and the experiment E94-107 in Hall A.
\end{abstract}

The spectroscopy of hypernuclei with electron beams is now an indispensable part of strangeness nuclear physics and the established co-operation between the two operating electron beam facilities will further stimulate the field.

PACS: 13.60.Le, 25.30.Rw, 21.80.+a

XLIX International Winter Meeting on Nuclear Physics, BORMIO2011

January 24-28, 2011

Bormio, Italy

\footnotetext{
*Speaker.
} 


\section{Introduction}

A very interesting phenomenon in nuclear physics is the existence of nuclei containing hyperons. Some hyperons decay only weakly and as they do not suffer from Pauli blocking by other nucleons they live long enough in the nuclear cores to become bound. When a hyperon, specifically a $\Lambda$-hyperon, replaces one of the nucleons in the nucleus, the original nuclear structure changes to a system composed by the hyperon and the core of the remaining nucleons. Thus, a $\Lambda$-hyperon bound to a nuclear core, forming a hypernucleus, is a unique probe of the interior of hadronic many-body systems.
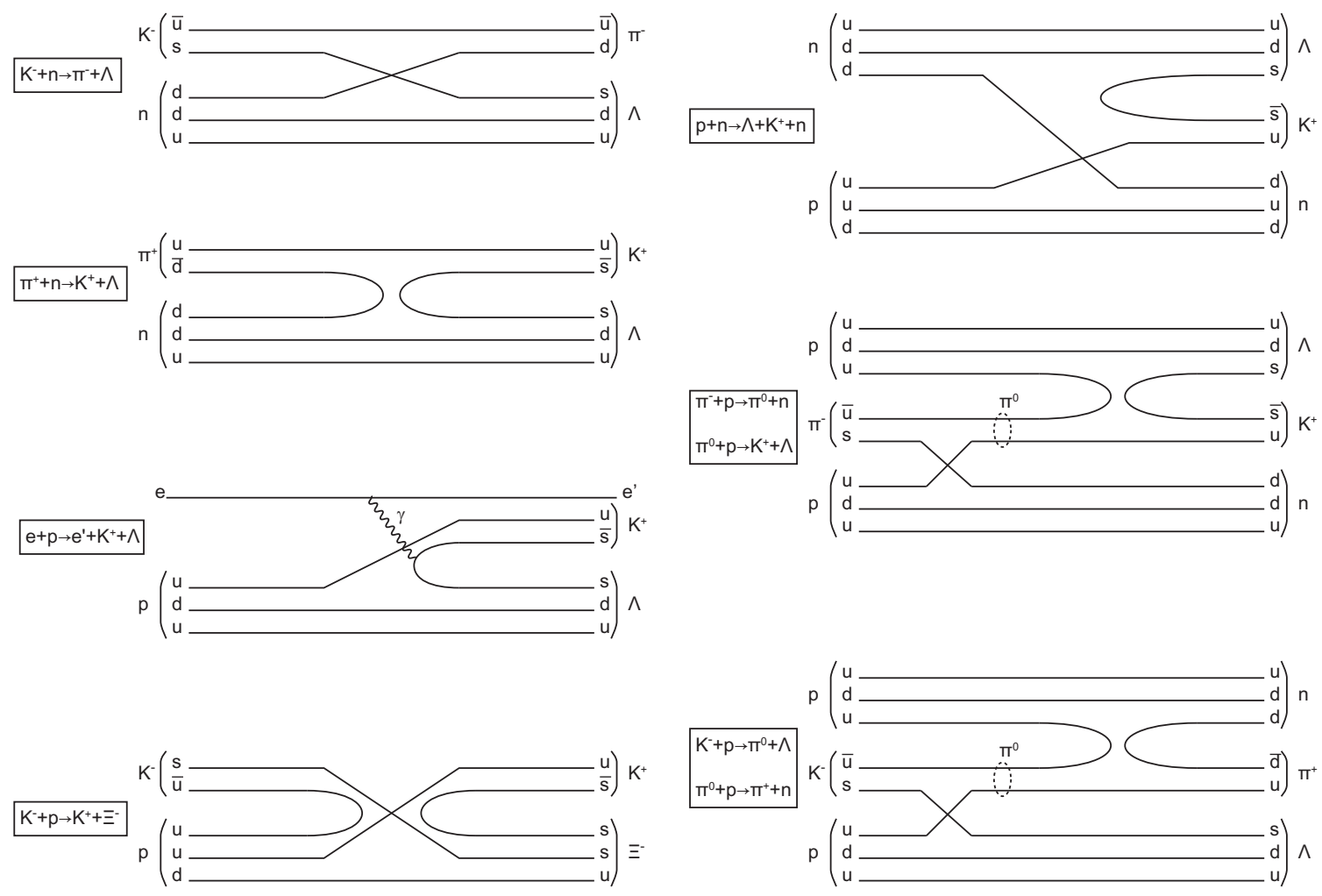

Figure 1: Quark flow diagrams of possible hypernuclei production mechanisms. Kaon and pion induced single $\Lambda$-hypernuclei production reactions are shown top left, the corresponding electro-production reaction below, and $\Xi$-production, leading to double strange hypernuclei, bottom left. A possible hadro-production reaction is shown top right, double charge exchange reactions to produce neutron richer hypernuclei are depicted below.

The production and detection of hypernuclei at accelerators can proceed with different beams using different experimental techniques. In Fig. 1 quark flow diagrams of the most relevant reactions mechanisms are depicted to visualize the processes on the quark level. $\Lambda$-hypernuclei, being the most stable hypernuclei, have been extensively studied during the past 60 years [1]. Mesoninduced reactions have been predominantly utilized for these studies, the spectroscopy with electron beams was realized only recently. For light nuclear targets the different reactions populate distinct isotopic regions of hypernuclei without overlap, demonstrating the complementarity of the various production mechanisms and the need to study hypernuclei with different reactions. Until 
now, around 40 different $\Lambda$-hypernuclear isotopes have been identified. In reaction spectroscopy ground and excited hypernuclear states can be identified by a missing mass analysis of the incident beam and the associated meson. Since these reactions require stable target nuclei, hypernuclei accessible by these reactions are limited. Even though an increasing number of new experiments are now being performed in hypernuclear spectroscopy, our knowledge on hypernuclear binding energies and detailed spectroscopic levels refer mostly to light ( $s$ - and $p$-shell) hypernuclei. Many more isotopes are accessible through hypernuclear fragmentation reactions induced $e$.g. by heavyion beams.

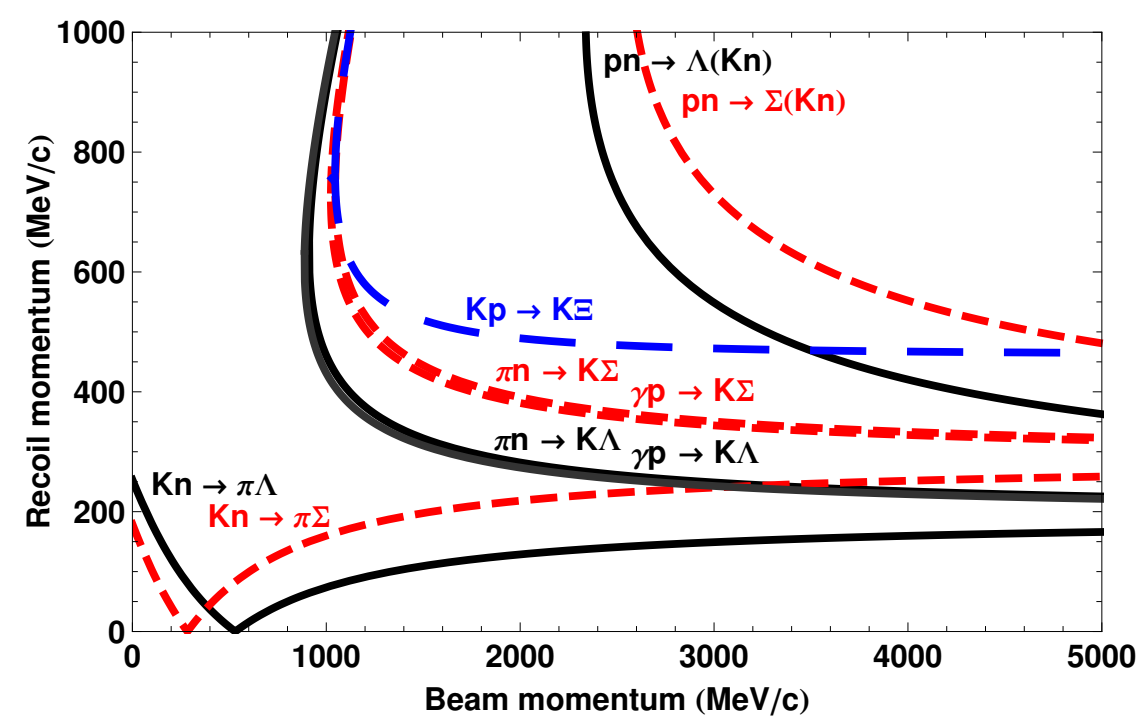

Figure 2: Minimum recoil momentum of $\Lambda, \Sigma^{0}$, and $\Xi^{-}$-hyperons in $\gamma p \rightarrow K^{+} Y$ photo-production, in $\pi^{+} n \rightarrow K^{+} Y$ pion induced production, in $K^{-} n \rightarrow \pi^{-} Y$ kaon induced production, in $p n \rightarrow\left(K^{+} n\right) Y$ hadron induced production, as well as in $K^{-} p \rightarrow \Xi^{-} K^{+}$charge exchange reaction. In the electromagnetic production the recoil momentum is $q_{Y} \sim 250-300 \mathrm{MeV} / c$ for larger photon energies.

In order to form a hypernucleus, the hyperon produced in the reaction has to get bound by the core nucleus. This formation depends very much on the transferred momentum to the hyperon. If the momentum transfer is large compared with typical nuclear Fermi momenta, the hyperon will emerge from the nucleus. In Fig. 2 the minimum momentum transfer to the hyperon $Y=\left(\Lambda, \Sigma^{0}, \Xi^{-}\right)$in the reaction $(i, f) Y$ is shown as a function of the projectile momentum $p_{i}$ at emission angles $\theta_{f}=0^{\circ}$ in the laboratory frame. With the exception of the $\left(K^{-}, \pi^{ \pm}\right)$process the reactions are endoenergetic and the hyperon cannot be produced at rest. Namely in the electromagnetic production the recoil momentum, $q_{Y}$, decreases strongly near the production threshold as the projectile momentum, i.e. the photon energy, increases but does not fall below $q_{Y} \sim 250$ $300 \mathrm{MeV} / c$ in the $K \Lambda$ channel. In this kinematic situation the hyperon is produced with a significant probability in a quasi-free process.

\section{Electro-Production of Strange Nuclear Systems}

Technical and methodical development made it possible that today strange systems can also be produced using electromagnetic processes. A new era started with the application of high-energy, 


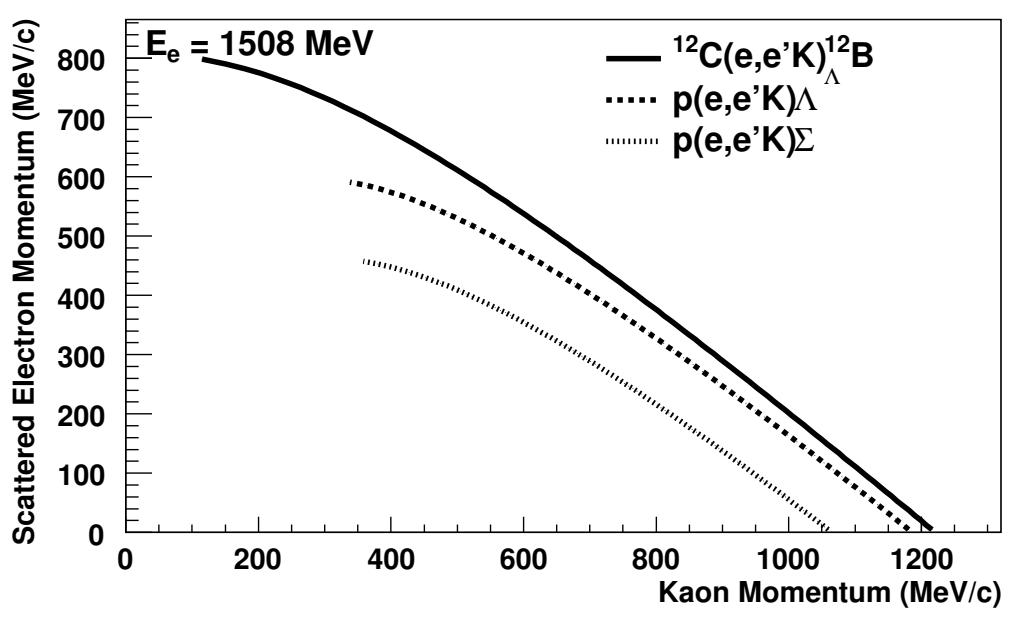

Figure 3: Kinematic correlation between scattered electron momentum and maximum kaon momentum in elementary $p\left(e, e^{\prime} K^{+}\right) Y$ and ${ }_{\Lambda}^{12} \mathrm{~B}$ hypernuclear reactions for an electron beam energy of $1508 \mathrm{MeV}$. For the reaction spectroscopy two spectrometers or spectrometer arms with a fixed central momentum relation are needed for the coincident detection of the two outgoing particles.

high-intensity continuous electron beams available at two places worldwide: MAMI at Mainz, Germany, and Jefferson Lab (JLab), Virginia. These beams are the ideal probes to study the hadronic structure of nuclei and nucleons. The machines are capable of up to $100 \mu \mathrm{A}$ beam current, with $100 \%$ duty factor. In the experimental halls the facilities utilize high-resolution large-acceptance focusing spectrometers, and high-resolution detectors with fast electronics. Magnetic spectrometers with high resolving powers tend to be long, which makes them hard to use for measurements of particles as short-lived as kaons. Consequently, for the detection of kaons dedicated strangeness spectrometers with short particle orbits were developed. These instruments make measurements of fairly low cross-sections possible which lead to improved statistical and systematic errors relative to earlier facilities.

In a typical two-arm strangeness electro-production experiment a charged kaon is detected in coincidence with the scattered electron and the recoiling hadronic system remains unobserved. As an example for the electro-production kinematics at a typical electron beam energy of $1508 \mathrm{MeV}$ the correlation between the scattered electron momentum and the maximum kaon momentum is shown in Fig. 3. The correlation has been calculated for the elementary $\Lambda$ - and $\Sigma$-hyperon production off the proton and for the ${ }_{\Lambda}^{12} \mathrm{~B}$ hypernuclei production off a carbon target. For the reaction spectroscopy two spectrometers or spectrometer arms with a fixed central momentum relation are needed for the coincident detection of the two outgoing particles.

The electron beams have excellent spatial and energy definitions, and targets can be physically small and thin $\left(10-50 \mathrm{mg} / \mathrm{cm}^{2}\right)$ which is of great practical advantage in the study of many isotopes. The $p\left(e, e^{\prime}, K^{+}\right)$reaction, in contrast to most meson-induced reactions, produces hypernuclei by converting a proton into a hyperon and transfers a large momentum to a hypernucleus. The small cross-section for the reaction, $\sigma \sim 140 \mathrm{nb} / \mathrm{sr}$ on a ${ }^{12} \mathrm{C}$ target, compared to strangeness exchange $\mathrm{n}\left(K^{-}, \pi^{-}\right) \Lambda$ or to associated production $\mathrm{n}\left(\pi^{+}, K^{+}\right) \Lambda$ is well compensated by the available high electron beam intensities. 
In electro-production the angular distribution of kaons associated with a given hypernuclear state is sensitive to the $\Lambda$ wave function inside the nucleus [2,3]. A hypernuclear $\Lambda$-probe samples the nuclear core where there is little direct information on the single particle structure. In addition, high resolution spectroscopic studies of hypernuclei can be performed in order to provide the most valuable experimental information on the $\Lambda$ dynamics in nuclei. The electro-production process has the unique characteristic of providing large amplitudes for the population of spin-flip hypernuclear states with unnatural parities [4]. For calculating hypernuclear production cross-sections, precise data from kaon electro-production processes are urgently required. The elementary amplitude serves as the basic input, which determines the accuracy of predictions for hypernuclei $[5,6]$.

The electron beam at JLab permitted the first successful $\left(e, e^{\prime} K^{+}\right)$spectroscopy measurements [7] as the necessary beam energy of above $1 \mathrm{GeV}$ was available many years earlier than at MAMI. The study of single $\Lambda$-hypernuclei in light targets at MAMI was first proposed by J. Pochodzalla [8]. With the completion of the fourth accelerator stage, called MAMI-C, in 2007, the threshold beam energy for associated strangeness production off protons was reached.

\subsection{Program at Jefferson Lab}

In 2000, first electro-production experiments on carbon targets were performed at Jefferson Lab using the HyperNuclear Spectrometer System (HNSS) in Hall C demonstrating the feasibility of reaction spectroscopy of ${ }_{\Lambda}^{12} \mathrm{~B}$ hypernuclei [7,9]. A split-pole type spectrometer (called Enge after its designer) was used as the scattered electron spectrometer. Kaons were measured by the Short Orbit Spectrometer (SOS) of Hall C. In these pioneering experiments a missing mass resolution of $3.5 \mathrm{MeV}$ (FWHM) was reported which was dominated by the kinematic broadening due to the $\Lambda$ recoil [7]. These measurements were also limited in statistics, but triggered new activities at Jefferson Lab. Experiment E94-107 was performed in 2004-5 in Hall A. In the hypernuclear missing-mass spectrum the experiment achieved very good energy resolution (670 keV FWHM) by exploiting the characteristics of the High Resolution Spectrometer (HRS) pair and the good beam quality available at JLab. The spectrometers were used with the addition of a pair of septum magnets to reach the desired small angles [10]. Experiment 94-107 obtained, in addition to the high resolution ${ }_{\Lambda}^{12} \mathrm{~B}$ spectrum, the excitation spectra for ${ }_{\Lambda}^{9} \mathrm{Li}$ and ${ }_{\Lambda}^{16} \mathrm{~N}$. Experiment E01-011 was performed in 2005 in Hall C and provided excitation spectra for ${ }_{\Lambda}^{7} \mathrm{He}$ and ${ }_{\Lambda}^{28} \mathrm{Al}$ [11]. The experimental geometry included a new high resolution kaon spectrometer and implemented a "tilt method" for the electron spectrometer to increase the hypernuclear yield and the signal to noise ratio by avoiding the electron background at zero degrees.

The third generation experiment E05-115 in Hall C employed two spectrometers dedicated to hypernuclear physics: the High-resolution Electron Spectrometer (HES) and the the Highresolution Kaon Spectrometer (HKS). In 2009, E05-115 took data for elementary hyperon electroproduction and measured the excitation spectra of ${ }_{\Lambda}^{7} \mathrm{He},{ }_{\Lambda}^{9} \mathrm{Li},{ }_{\Lambda}^{10} \mathrm{Be},{ }_{\Lambda}^{12} \mathrm{~B}$, and ${ }_{\Lambda}^{52} \mathrm{~V}$ hypernuclei. New data for vanadium are expected to provide information on the behaviour of a $\Lambda$-hyperon in a nucleus and on the characteristic structure of a medium-heavy hypernucleus. The target was chosen considering the expected clean spectrum that demonstrates major shell structure.

In this latest experimental configuration, a splitter magnet was installed downstream of the target in order to measure electrons and kaons at very forward angles. Scattered electrons and positive kaons at very forward angles were deflected to the opposite directions and guided to each 
spectrometer. After optimization of both spectrometers, the newly designed beam splitter, and the pre-target beam chicane, the experiment has collected a large statistic sample of hypernuclear events. The analysis is ongoing with a particular emphasis of determining precision absolute hypernuclear masses.

The experimental techniques for the electro-production of hypernuclei is now fully developed. In fact, the high precision of electron beams have considerably improved the quality of experimental data.

\subsection{Program at MAMI}

The spectrometer facility at MAMI operated by the A1 collaboration offers the opportunity to electron scattering reactions on various solid-state or liquid cryogenic targets in a wide range of kinematics. Since the 1990s the spectrometer facility consisted of three vertically deflecting magnetic spectrometers (SpekA, SpekB, and SpekC) rotating around a common pivot and with deflection angles of $90^{\circ}$ to $120^{\circ}$ and high momentum resolution [12].

The KAOS spectrometer was considered to be ideal for the strangeness electro-production programme at MAMI. The spectrometer is a very compact instrument suitable especially for the detection of kaons [13]. Fig. 4 shows the survival probability for kaons to be detected in the high resolution spectrometers SpekA, SpekB, and SpekC as well as in the KAOS spectrometer. The momentum acceptance of the high resolution spectrometers is limited by the indicated maximum momenta. It is easily seen that the detection of kaons is not effective with the high-resolution spectrometers due to the short life-time of the charged kaons and the long flight path through the spectrometers.

The installation of the spectrometer started in mid 2006. During the years 2007-8 the in-beam commissioning of the KAOS spectrometer at MAMI has been achieved with a total of five beamtimes in February, March, September, October, and November. The spectrometer was officially inaugurated with a ceremony on 5 June 2009.

The hypernuclear reaction spectroscopy experiments at MAMI require the detection of both, the associated kaon and the scattered electron, at very forward laboratory angles. To accomplish this both particles will be detected simultaneously by the KAOS spectrometer which was instrumented in two arms, to either side of the main dipole. In February 2009 the spectrometer was set up for the first time comprising two arms, with a new vacuum chamber extending the existing vacuum system of the dipole to the electron arm focal plane region. The electron arm instrumentation consists of two vertical planes of 18432 fibres. Detectors and electronics for the 4608 read-out and level-1 trigger channels have been installed and tested, beam-tests started in 2009 and were completed in 2010 .

When operating the KAOS spectrometer zero degree relative to the incident beam the primary beam will pass through the dipole field of the spectrometer after impinging on the target and will be bent away from its original axis. Thus, a magnetic chicane comprising compensating magnets was installed. The beam angle at the target can be varied by adjusting the strength of the magnetic fields in both chicane magnets and the position of the second magnet. The chicane was brought into operation in February 2011. Fig. 5 shows the spectrometer facility at MAMI as it is operated by the A1 collaboration with the pre-target beam chicane. The KAOS spectrometer complements 


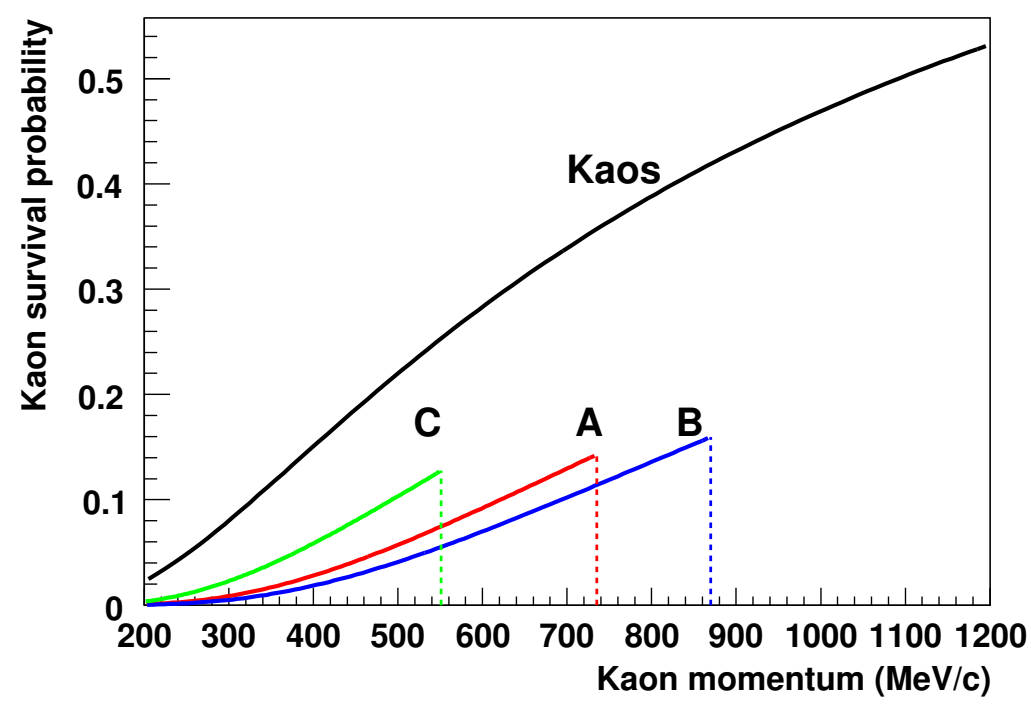

Figure 4: Survival probability for kaons to be detected in the high resolution spectrometers A, B, and C and in the KAOS spectrometer. The momentum acceptance of the high resolution spectrometers is limited by the indicated maximum momenta.

the existing spectrometers and is the instrument of central importance for strangeness reactions on the proton or light nuclei.

It is known from the emulsion experiments, see e.g. [14], that many different hypernuclei, including proton or neutron rich ones, can be produced as $\Lambda$-hyperfragments. Precision pion spectroscopy of the hyperfragments will improve our knowledge on their binding energies by about one order of magnitude, thus allowing for the first time precision studies of the charge symmetry breaking in hypernuclei. One good example is the difference in binding energies, $B_{\Lambda}$, of ${ }_{\Lambda}^{4} \mathrm{H}$ and ${ }_{\Lambda}^{4} \mathrm{He}$ hypernuclei, which show the charge symmetry breaking in $Y N$ interactions. Since, so far, the values of $B_{\Lambda}$ for light hypernuclei were almost determined only by emulsion, an accurate check on the emulsion values of $B_{\Lambda}$ is important. The search for isomeric low lying states and the study of the drip line limit on $\Lambda$-hypernuclei with small production probabilities, such as heavy hyperhydrogen ${ }_{\Lambda}^{6} \mathrm{H},{ }_{\Lambda}^{7} \mathrm{H}$ and ${ }_{\Lambda}^{8} \mathrm{H}$ or extremely neutron rich ${ }_{\Lambda}^{11} \mathrm{Li}$ and ${ }_{\Lambda}^{12} \mathrm{Li}$ are important future extensions of the program.

The first hypernuclei experiments on pion decay spectroscopy to be performed with the KAOS spectrometer as strangeness tagger are scheduled for Summer 2011. The produced hypernuclei, predominantly in forward direction, will be stopped in the target and decay after some $200 \mathrm{ps}$ inside of the target. Decay pions have a discrete momentum lying in the range $\leq 133 \mathrm{MeV} / \mathrm{c}$ and will leave the target. For the measurements of momentum and outgoing angles of these monochromatic pions spectrometer $\mathrm{C}$ will be used. The central optical axis of the spectrometer will be normal to the target plane so that the target straggling energy loss uncertainty of the decayed pions will be minimized. As target material natural lithium $\left(7.5 \%{ }^{6} \mathrm{Li}, 92.5 \%{ }^{7} \mathrm{Li}\right)$ with $0.38 \mathrm{~mm}\left(=20.3 \mathrm{mg} / \mathrm{cm}^{2}\right)$ thickness and beryllium $\left({ }^{9} \mathrm{Be}\right)$ with $0.125 \mathrm{~mm}\left(=23.1 \mathrm{mg} / \mathrm{cm}^{2}\right)$ thickness are installed in a watercooled target ladder, additional Be targets are being prepared. A new photon beam-dump was developed to reduce the observed high background rate in the detectors. Positively charged kaons 

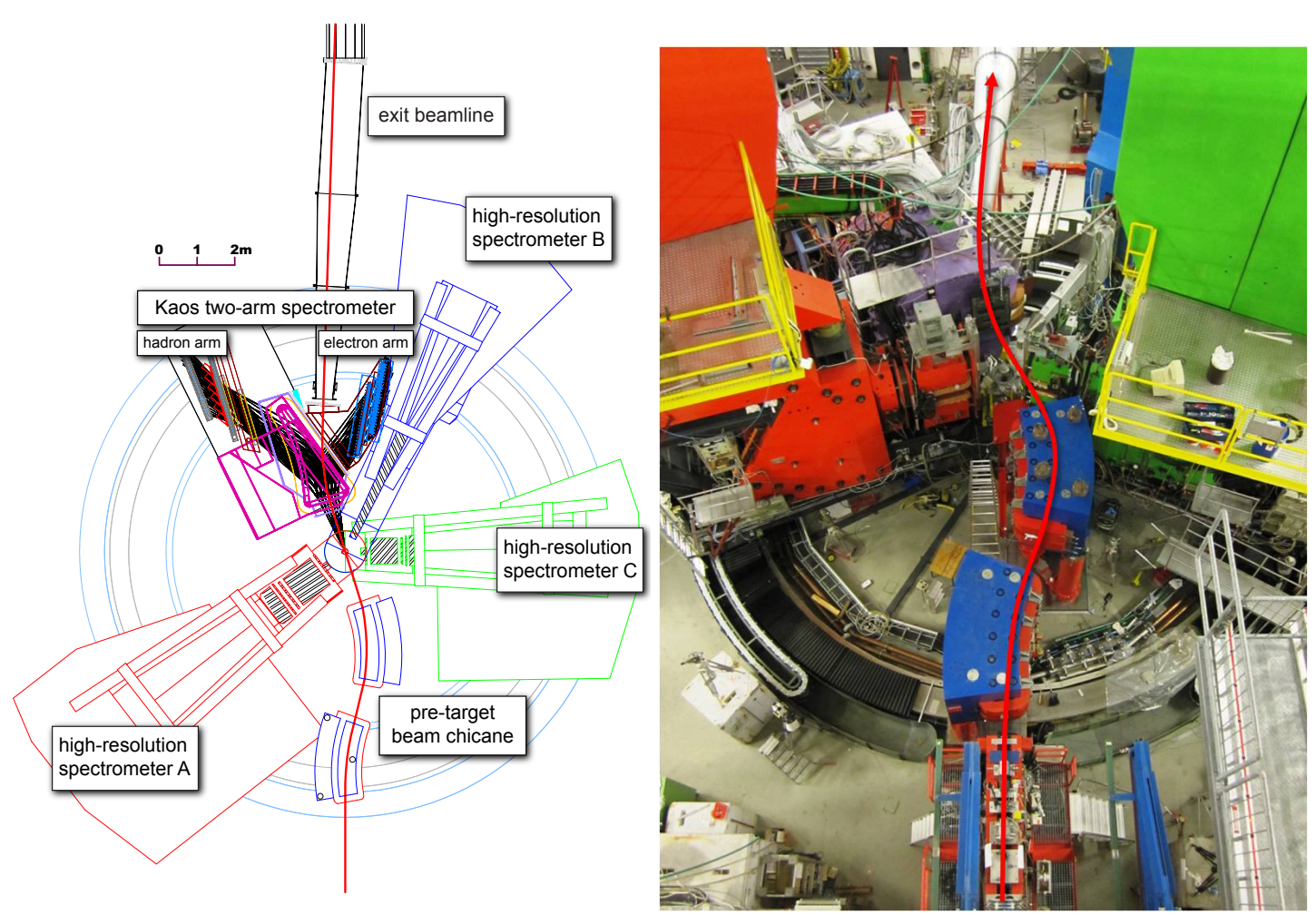

Figure 5: The spectrometer facility at MAMI operated by the A1 collaboration. The high resolution spectrometers A, B, and C are complemented in forward direction by the KAOS spectrometer. A new beam-line with a pre-target beam chicane was installed to operate the KAOS spectrometer around zero degree relative to the incident beam, maximising hypernuclear production rates at nuclear targets. Charged particles will be detected on both sides of the beam exit. In the schematics ray-traced trajectories are shown by full lines and the instrumentation of the two spectrometer arms is indicated.

will be detected by the KAOS spectrometer. Kaon identification is based on specific energy-loss and time-of-flight, pion identification on a missing signal in a gas Cherenkov counter. The KAOS spectrometer was upgraded to improve its kaon identification power by

- a new scintillator wall as the old walls were aged and showed a strong absorption of light. Furthermore, sizeable gaps in acceptance of the old scintillator walls existed. The new wall consists of 30 paddles and is read out by 60 PMTs.

- a new aerogel Cherenkov detector for pion suppression. This detector has a large sensitive area to match spectrometer acceptance and is slim enough to fit in the slot between the two scintillator walls. The new detector consists of 6 segments and is read out by 12 five-inch PMTs.

Prototypes and segments of both detectors were extensively in-beam tested. The high energy definition of the MAMI beam, the versatile high resolution spectrometer $\mathrm{C}$ for the pion detection and the large acceptance of the KAOS spectrometer for kaon tagging will be essential for this experiment. 


\section{Conclusions}

The spectroscopy of hypernuclei with electron beams, realized either as reaction spectroscopy or as decay particle spectroscopy, is now an indispensable part of strangeness nuclear physics and the established co-operation between the two operating electron beam facilities will further stimulate the field.

\section{References}

[1] O. Hashimoto, H. Tamura, Prog. Part. Nucl. Phys. 57, 564 (2006)

[2] S. Shinmura, Prog. Theor. Phys. 92, 571 (1994)

[3] C. Bennhold, H. Haberzettl, T. Mart, A new resonance in $K^{+} \Lambda$ electroproduction: The $D_{13}(1895)$ and its electromagnetic form factors, arXiv:nucl-th/9909022 (1999)

[4] T. Motoba, M. Sotona, K. Itonaga, Prog. Theor. Phys. Suppl. 117, 123 (1994)

[5] T. Motoba, P. Bydžovský, M. Sotona, K. Itonaga, K. Ogawa, O. Hashimoto, Prospects of photoproduction of medium-heavy hypernuclei, in Proceedings of the International Symposium on Electrophotoproduction of Strangeness on Nucleons and Nuclei, Sendai, Japan, 16-18 June 2003, edited by K. Maeda, H. Tamura, S.N. Nakamura, O. Hashimoto (World Scientific, Singapore, 2004), pp. 221-232

[6] P. Bydžovský, T. Mart, Phys. Rev. C 76, 065202 (2007)

[7] T. Miyoshi et al. (HNSS), Phys. Rev. Lett. 90(23), 232502 (2000)

[8] J. Pochodzalla, Nucl. Phys. A 754, 430c (2005)

[9] L. Yuan et al. (HNSS/E93-018), Phys. Rev. C 73, 044607 (2006)

[10] J.J. LeRose et al. (Hall A), Nucl. Phys. A 804, 116 (2008)

[11] S.N. Nakamura, Future hypernuclear experiments at JLab, in Proceedings of the IX International Conference on Hypernuclear and Strange Particle Physics, Johannes Gutenberg-Universität, Mainz, 10-14 October 2006, edited by J. Pochodzalla, Th. Walcher (Springer, Berlin/Heidelberg, 2007), pp. $51-56$

[12] K.I. Blomqvist et al. (A1), Nucl. Instr. and Meth. Phys. Res. A 403(3), 263 (1998)

[13] P. Senger et al. (KaoS), Nucl. Instr. and Meth. Phys. Res. A 327(2-3), 393 (1993)

[14] D.H. Davis, Nucl. Phys. A 754, 3c (2005) 